\title{
Serum lipids and brain-derived neurotrophic factor in a cross-section of male students with symptoms of depression at a university in Iran: an observational study
}

Ramin Tavakoli', Hamid Yaghooti ${ }^{2 * *}$, Robab Daghagheleh ${ }^{3}$, Rohollah Yousofi', Parisa Rahimifar ${ }^{1}$

\begin{abstract}
Background: Depression is a neuroprogressive disorder that is characterized by neurotransmitter derangement and decreased neurogenesis and neurotrophic factors including brain-derived neurotrophic factor (BDNF).

Objectives: To determine the lipid profiles and BDNF levels in university students at an institution in Iran and association of these factors with Beck Depression Inventory (BDI) scores.

Methods: We conducted an observational study of a cross-section of male students at the Ahvaz Jundishapur University of Medical Sciences in Iran. For each of the 100 participants, a BDI score was obtained and serum levels of BDNF were measured by enzyme-linked immunosorbent assay. Levels of serum lipids, including cholesterol, triglycerides, lowdensity lipoprotein (LDL), and high-density lipoprotein (HDL), were measured using a biochemical analyzer. Castelli's risk index type I (CRI-I), Castelli's risk index type II (CRI-II), CRI-I = TG/HDL-C and CRI-II = LDL-cholesterol/ HDL-cholesterol, and atherogenic index of plasma (AIP), AIP $=\log$ (triglycerides/HDL-cholesterol), were calculated. Results: Based on BDI scores, lower levels of BDNF, triglycerides, cholesterol, and HDL, but higher levels of LDL were found in participants with higher BDI scores. CRI-I was also increased in participants with depression.

Conclusion: The levels of BDNF and lipid factors are associated with the severity of depression in Iranian male university students. Deranged levels of BDNF and lipids may predispose depressed students to cardiovascular diseases.
\end{abstract}

Keywords: BDNF, Beck Depression Inventory, depression, lipid profile

Depression is a neuroprogressive disorder that is characterized by decreased neurogenesis and neurotrophic factors, including brain-derived neurotrophic factor (BDNF). Depression is associated with derangement of neurotransmitters such as serotonin, dopamine, noradrenaline, melatonin, and glutamate in the central nervous system (CNS) [1,2]. BDNF and lipid factors are potential biomarkers for depression. BDNF acts as a regulator of neuron growth. Various functions, such as regulating neuronal development, survival, durability, and plasticity of neurons, have been identified for BDNF [3].

*Correspondence to: Hamid Yaghooti, Department of Medical Laboratory Sciences, Hyperlipidemia Research Center, School of Allied Medical Sciences, Ahvaz Jundishapur University of Medical Sciences, Ahvaz 61357-15794, Iran, e-mail: yaghooti-h@ajums.ac.ir 'Department of Biochemistry, School of Medicine, Ahvaz Jundishapur University of Medical Sciences, Ahvaz 61357-15794, Iran 2Department of Medical Laboratory Sciences, Hyperlipidemia Research Center, School of Allied Medical Sciences, Ahvaz Jundishapur University of Medical Sciences, Ahvaz 61357-15794, Iran

${ }^{3}$ Counseling Office, School of Allied Medical Sciences, Ahvaz Jundishapur University of Medical Sciences, Ahvaz 61357-15794, Iran O Open Access. ๑ 2017 Ramin Tavakoli et al., published by Sciendo. @) BY-Nc-ND This work is licensed under the Creative Commons Attribution NonCommercial-NoDerivatives 4.0 License. 
Moreover, BDNF is involved in the regulation of neurotransmitters and monoamine synthesis [4]. Decreased BDNF has been reported in individuals suffering from severe depression, and antidepressant treatment restored the decreased BDNF levels toward normal in these patients [5].

Depression is associated with metabolic changes such as decreased cholesterol and high-density lipoprotein cholesterol (HDL-C) levels, and increased triglyceride level [6-8]. Lipid disorders lead to increased atherogenic potential. Increased Castelli's risk index type I (CRI-I), Castelli's risk index type II (CRI-II), and atherogenic index of plasma (AIP) are appropriate markers for evaluating the risk of cardiovascular disease [9].

Collegiate life is a stressful period because of the presence of multiple stressors. A high prevalence of depression among university students of up to $37 \%$ has been reported [10]. It is important to diagnose the disorder early and manage and treat the suffering students. Treatment can avoid serious outcomes of this condition such as suicide.

In the present study, we evaluated depression in a crosssection of Iranian university students using the Beck Depression Inventory (BDI) score. We also determined lipid profile and BDNF levels in the students and analyzed association of these factors with depression and BDI scores in the convenience sample. We aimed to study how AIP, CRI-I and CRI-II, and lipid profile were different in the depressed students. We hypothesized that metabolic changes were associated with decreased BDNF.

\section{Methods}

\section{Participants}

The study protocol was approved by the institutional ethics committee of the Ahvaz Jundishapur University of Medical Sciences (approval no. IR.AJUMS.REC.1395.76) and was conducted in accordance with the ethical standards specified in the 1964 Declaration of Helsinki and its contemporary amendments. Documented informed consent was obtained from all participants before including them in the study. After approval of the protocol, we conducted an observational study of a cross-section of male students at the Ahvaz Jundishapur University of Medical Sciences. A convenience sample of 100 male students aged from 20 to 27 years comprised healthy male students and students with depression who were diagnosed based on the BDI score. These students were not receiving any medication, special diet, or any previous psychiatric treatment. Students with a very low or high body mass index (BMI) $\left(<20 \mathrm{~kg} / \mathrm{m}^{2}\right.$ or $\left.>30 \mathrm{~kg} / \mathrm{m}^{2}\right)$ or those who were consumers of tobacco or alcohol were excluded from the study. The students presented various levels of depression symptoms assessed using the BDI. The BDI is a multiple-choice selfreport inventory and one of the most widely used psychometric tests for measuring the severity of depression, being rooted in the patient's cognitive, affective, motivational, and somatic symptoms of depression. The long form of the BDI that we used contained 21 questions. Each item was rated on a 4-point (0-3) Likert-style scale. The maximum score in this form was 64, which indicated severe depression. The student participants were categorized into 4 groups including 30 students without any symptom of depression, 26 students with mild depression, 31 students with moderate depression, and 13 students with severe depression manifestations. Medical history, health investigation, and measurement of weight, height, BMI, systolic blood pressure (SBP), and diastolic blood pressure (DBP) were obtained.

\section{Biochemical measurements}

Following psychological evaluation and determining the BDI scores, a blood sample was drawn for biochemical measurements. Venous blood was collected from the participants after $12 \mathrm{~h}$ fasting and a low-fat diet for several days. Serum samples were separated following centrifugation of the blood. The BDNF level was measured using a commercial enzyme-linked immunosorbent assay kit (interassay coefficient of variation (CV) 4.3\%; Boster Bio). Levels of serum lipid profile, including cholesterol, triglycerides, low-density lipoprotein (LDL), and high-density lipoprotein (HDL), were measured using a biochemical analyzer (BT 300) with assay kits (Pars Azmoon Inc., Tehran, Iran). Interassay CVs of the measurements were $1.8 \%$ for triglycerides, $1.3 \%$ for cholesterol, and $2.5 \%$ for HDL. CRI-I = TG/HDL-C, CRI-II = LDL-cholesterol/HDL-C, and AIP $=\log$ (triglycerides/HDL-C) were calculated.

\section{Statistical analysis}

Quantitative data in groups with various levels of depression are presented as mean $\pm \mathrm{SD}$. The normality of the variables was evaluated using a Kolmogorov-Smirnov test. To determine the relationship between BDI scores and BDNF, triglycerides, cholesterol, LDL, and HDL levels; CRIs; and AIP, we conducted a multivariable regression adjusted for age and BMI. Biochemical variables were compared between groups using analysis of variance (ANOVA) followed by a Tukey post hoc multiple comparison because the variables in the 4 groups were distributed normally. $P<0.05$ was considered as significant. 
A sample size of 96.04 was calculated based on Cochran's sample size formula with an assumed power of $80 \%$ and rounded to 100 in the present study.

\section{Results}

Table 1 shows the demographic and laboratory data of the students. There were no significant differences in age, BMI, weight, DBP, or SBP between the groups of student participants with various levels of depression. Lower levels of BDNF were found in participants with higher levels of depression. Participants with moderate and severe depression showed significantly lower levels of BDNF than those without depression $(P<0.01$ adjusted for age and BMI). Measurements of lipids and lipoproteins in participants in the depression groups showed various profiles for triglycerides, cholesterol, LDL, and HDL when adjusted for age and BMI. The serum triglyceride level was lower in participants with depression. The differences between participants with severe and moderate depression were significant $(P<0.001)$. Similarly, levels of serum cholesterol and HDL were diminished in participants with depression. There were significantly lower levels of serum cholesterol and HDL in the participants with moderate and severe depression ( $P<0.001$ for both). By contrast, serum LDL levels were higher with increasing severity of depression. Significantly higher levels of LDL were found in participants with severe symptoms of depression compared with those in nondepressed participants $(P<0.001)$. CRI-I was significantly higher in the student participants with severe depression, but AIP index was significantly lower in those with severe depression. To study the association of the measured biochemical variables with the BDI scores, multivariate linear regression analysis adjusted for age and BMI was used. As shown in Table 2, there was a significant inverse correlation between the BDI scores and serum BDNF, triglycerides, cholesterol, and HDL levels $(P<0.001)$, whereas a significant positive correlation was found between the BDI scores and the levels of LDL ( $r=0.673, P<0.001)$.

Table 2. Multivariate regression of Beck Depression Inventory score with other clinical and biochemical variables

\begin{tabular}{lcc}
\hline Variable & $\boldsymbol{r}$ & $\boldsymbol{P}$ \\
\hline BDNF $(\mathrm{pg} / \mathrm{mL})$ & -0.828 & $<0.001$ \\
Triglycerides $(\mathrm{mg} / \mathrm{dL})$ & -0.369 & $<0.001$ \\
Cholesterol $(\mathrm{mg} / \mathrm{dL})$ & -0.626 & $<0.001$ \\
HDL $(\mathrm{mg} / \mathrm{dL})$ & -0.365 & $<0.001$ \\
LDL $(\mathrm{mg} / \mathrm{dL})$ & 0.673 & $<0.001$ \\
SBP $(\mathrm{mmHg})$ & -0.154 & 0.13 \\
DBP $(\mathrm{mmHg})$ & -0.156 & 0.14 \\
CRI-I & 0.560 & $<0.001$ \\
CRI-II & -0.174 & 0.08 \\
AIP & -0.245 & 0.015 \\
\hline
\end{tabular}

Multivariate model adjusted for age and body mass index AIP, atherogenic index of plasma; BDNF, brain-derived neurotrophic factor; CRI-I, Castelli's risk index type I; CRI-II, Castelli's risk index type II; DBP, diastolic blood pressure; HDL, high-density lipoprotein; LDL, lowdensity lipoprotein; SBP, systolic blood pressure

Table 1. Beck Depression Inventory scores and demographic and laboratory data of students with different levels of depression symptoms

\begin{tabular}{|c|c|c|c|c|c|}
\hline Variable & No depression $(n=30)$ & Mild $(n=26)$ & Moderate $(n=31)$ & Severe $(n=13)$ & $P$ \\
\hline BDI score & $7.5 \pm 4.4$ & $22.2 \pm 4.8$ & $39.5 \pm 3.9$ & $50.6 \pm 3.2$ & $<0.001$ \\
\hline BDNF (pg/mL) & $935 \pm 363.4$ & $533 \pm 106.5$ & $264 \pm 84.3$ & $69 \pm 22.8$ & $<0.001$ \\
\hline Triglycerides (mg/dL) & $234 \pm 192.6$ & $152 \pm 38.4$ & $146 \pm 19.9$ & $94 \pm 11.4$ & $<0.001$ \\
\hline Cholesterol (mg/dL) & $196 \pm 44.6$ & $156 \pm 41.9$ & $126 \pm 23.0$ & $110 \pm 5.8$ & $<0.001$ \\
\hline $\mathrm{HDL}(\mathrm{mg} / \mathrm{dL})$ & $37.8 \pm 4.4$ & $37.7 \pm 5.3$ & $35.5 \pm 5.4$ & $29.8 \pm 3.3$ & $<0.001$ \\
\hline LDL (mg/dL) & $79.2 \pm 9.3$ & $89.3 \pm 7.4$ & $94.3 \pm 8.5$ & $106.1 \pm 8.2$ & $<0.001$ \\
\hline $\mathrm{DBP}(\mathrm{mmHg})$ & $80.8 \pm 7.9$ & $80.2 \pm 8.1$ & $80.5 \pm 7.6$ & $73.8 \pm 9.4$ & 0.10 \\
\hline $\mathrm{SBP}(\mathrm{mmHg})$ & $125.6 \pm 13.6$ & $123.1 \pm 12.9$ & $123.9 \pm 11.5$ & $116.3 \pm 9.5$ & 0.25 \\
\hline CRI-I & $0.058 \pm 0.017$ & $0.068 \pm 0.024$ & $0.081 \pm 0.026$ & $0.123 \pm 0.022$ & $<0.001$ \\
\hline AIP & $0.168 \pm 0.112$ & $0.116 \pm 0.051$ & $0.124 \pm 0.043$ & $0.110 \pm 0.026$ & 0.02 \\
\hline CRI-II & $0.134 \pm 0.042$ & $0.120 \pm 0.060$ & $0.107 \pm 0.036$ & $0.127 \pm 0.024$ & 0.13 \\
\hline Age (years) & $24.2 \pm 2.5$ & $24.2 \pm 2.2$ & $24.1 \pm 2.8$ & $23.1 \pm 2.5$ & 0.58 \\
\hline BMI $\left(\mathrm{kg} / \mathrm{m}^{2}\right)$ & $25.0 \pm 4.2$ & $25.4 \pm 4.5$ & $25.9 \pm 4.0$ & $23.8 \pm 3.7$ & 0.48 \\
\hline Weight (kg) & $73.1 \pm 11.8$ & $74.7 \pm 10.6$ & $77.3 \pm 9.1$ & $71.7 \pm 11.0$ & 0.31 \\
\hline Height $(\mathrm{cm})$ & $171 \pm 5$ & $172 \pm 7$ & $173 \pm 10$ & $174 \pm 10$ & 0.58 \\
\hline
\end{tabular}

AIP, atherogenic index of plasma; BDI, Beck Depression Inventory; BDNF, brain-derived neurotrophic factor; BMI, body mass index; CRI-I, Castelli's risk index type I; CRI-II, Castelli's risk index type II; DBP, diastolic blood pressure; HDL, high-density lipoprotein; LDL, low-density lipoprotein; SBP, systolic blood pressure 


\section{Discussion}

In the present study of a cross-section of male students at Ahvaz Jundishapur University of Medical Sciences in Iran, we found that the levels of triglycerides, cholesterol, HDL, and BDNF were lower in student participants with higher scores for depression according to their BDI score, whereas serum levels of LDL were higher in those with higher scores for depression. These findings suggest that greater attenuation of the BDNF level is associated with more severe depression. These findings are consistent with previous reports that the serum level of BDNF in people with symptoms of depression is lower than it is in normal individuals [3-5]. The administration of antidepressant drugs increases the serum levels of BDNF and alleviates symptoms of depression [11, 12]. Cholesterol is found at high levels in the CNS and plays various structural and functional roles in neurons. In the CNS, the membrane phospholipid bilayer is enriched with cholesterol, which is related to the blood cholesterol [13]. Changes in blood cholesterol can affect the fluidity and function of lipid membranes in the brain. Because serotonin receptors are located in the phospholipid membrane, deviations from the normal composition of membrane cholesterol can cause altered serotonin function and neural disturbances. Several lines of evidence have shown that cholesterol is a crucial component of the synaptic structure and function. Freeze fracture electron microscopy and biochemical studies have revealed that cholesterol is enriched in presynaptic terminals $[14,15]$. Levels of both BDNF and cholesterol dramatically increase during cortical development, indicating that BDNF may regulate cholesterol biosynthesis in the brain [16] and may elicit cholesterol biosynthesis for the development of presynaptic functions in the CNS [17]. BDNF upregulates mRNA levels for enzymes involved in cholesterol biosynthesis. At least 20 enzymes are involved in cholesterol biosynthesis, and BDNF increases the transcription of genes encoding these enzymes in neurons [18]. BDNF promotes cholesterol levels to regulate the availability of synaptic vesicles via a direct interaction with synaptophysin and synapse development [19].

Serum cholesterol and HDL levels in people who have committed suicide are lower than those in normal individuals. This finding supports the significant association between lipid metabolic factors and psychiatric disorders [20]. In a study of psychiatric patients, reduction in serum cholesterol was found to be a risk factor for suicidal behavior [21]. However, others could not show a positive and significant relationship between serum cholesterol and depression [22]. This is by contrast with the findings of Huang et al. who showed a significant positive relationship between decreased serum cholesterol and depression in both sexes and in all age groups [23].
There was a negative correlation between BDI scores and HDL in our present study. These findings are consistent with those of previous studies showing decreased HDL levels in depressed individuals [6, 7]. By contrast, Baek et al. reported that high HDL levels were associated with a recent suicide attempt in patients with a major depressive disorder [24]. There are also some studies that indicate a negative relationship between triglycerides and suicide ideation [24, 25]. In contrast to our findings, in these other studies, higher levels of triglycerides and LDL were found in depressed women [23] and older patients of either sex [26].

LDL-C levels were increased in our study, which resulted in elevated AIP and CRI-I and CRI-II. LDL-C is known as the most atherogenic lipid component. Our present results confirm the increased AIPs found in depressed individuals [23]. We propose that depression during university college life is a risk factor for cardiovascular diseases. This hypothesis is supported by the observation of higher morbidity of elderly patients with mood disorders. According to a cohort study, women with increased atherogenic indices also suffered from severe depressive disorders [27].

The present study has some limitations. Our participants were specifically male university students aged from 20 to 27 years at a single institution in Iran, and therefore, we cannot generalize our findings to all populations. Female students are kept separate from male students in the Islamic Republic of Iran and have separate dormitories and collegiate life. Female students were not available for interview or screening. A further limitation is the small convenience sample used in the study, in part, because of the difficulty in enrolling student participants who are depressed. No diagnosis of metabolic syndrome was made, and this would have assisted in interpretation of the lipid profiles.

\section{Conclusions}

This observational study of a cross-section of male university students at an institution in Iran showed that the serum levels of BDNF, triglycerides, cholesterol, HDL, and LDL were associated with the severity of depression. The depressed students presented higher atherogenic indices, which may predispose them to cardiovascular diseases. Further studies in larger and broader populations with various ethnicity and sex are warranted to determine the relationship between depression and BDNF, metabolic factors, and cardiovascular diseases risk.

Author contributions. HY and RD contributed substantially to the study design. RT and PR made substantial contributions to acquiring the data, and HY and RY substantially 
contributed to its analysis and interpretation. RT, RY, and PR drafted the manuscript, and $\mathrm{HY}$ and RD contributed substantially to its critical revision. All authors approved the final version submitted for publication and take responsibility for the statements made therein.

Acknowledgments. The authors thank the Student Research Committee of Ahvaz Jundishapur University of Medical Sciences, Ahvaz, Iran, for financial support of their work.

Conflicts of interest statement. The authors have each completed and submitted an ICMJE Uniform Disclosure Form for Potential Conflicts of Interest. None of the authors disclose any potential conflict of interest.

\section{References}

[1] Maletic V, Robinson M, Oakes T, Iyengar S, Ball S, Russell J. Neurobiology of depression: an integrated view of key findings. Int J Clin Pract. 2007; 61:2030-40.

[2] Maes M, Galecki P, Chang YS, Berk M. A review on the oxidative and nitrosative stress (O\&NS) pathways in major depression and their possible contribution to the (neuro) degenerative processes in that illness. Prog Neuropsychopharmacol Biol Psychiatry. 2011; 35:676-92.

[3] Chashmposh M, Shirali S, Ebrahimi E, Barari A. Correlation between hormonal and neurochemical changes and depression with menopausal STATUS: a systematic review. Women's Health Bull. 2015; 2:e25037. doi: 10.17795/whb-25037.

[4] Zuccato C, Cattaneo E. Brain-derived neurotrophic factor in neurodegenerative diseases. Nat Rev Neurol. 2009; 5:311-22.

[5] Lee B-H, Kim Y-K. The roles of BDNF in the pathophysiology of major depression and in antidepressant treatment. Psychiatry Invest. 2010; 7:231-5.

[6] Wang J, Jiang C, Chen L, Wu S, Lin J, Gao L, et al. A crosssectional study to investigate the correlation between depression comorbid with anxiety and serum lipid levels. Compr Psychiatry. 2016; 69:163-8.

[7] Lehto SM, Niskanen L, Tolmunen T, Hintikka J, Viinamäki H, Heiskanen T, et al. Low serum HDL-cholesterol levels are associated with long symptom duration in patients with major depressive disorder. Psychiatry Clin Neurosci. 2010; 64:279-83.

[8] Sagud M, Mihaljevic-Peles A, Pivac N, Jakovljevic M, Muck-Seler D. Lipid levels in female patients with affective disorders. Psychiatry Res. 2009; 168:218-21.

[9] Nunes SOV, de Melo LGP, de Castro MRP, Barbosa DS, Vargas $\mathrm{HO}$, Berk M, et al. Atherogenic index of plasma and atherogenic coefficient are increased in major depression and bipolar disorder, especially when comorbid with tobacco use disorder. J Affect Disord. 2015; 172:55-62.

[10] Jalilian F, Emdadi S, Karimi M, Barati M, Gharibnavaz H. Depression among collage students, the role of general self-efficacy and perceived social support. Sci J Hamadan Univ Med Sci. 2012; 18:60-6.
[11] Aydemir O, Deveci A, Taneli F. The effect of chronic antidepressant treatment on serum brain-derived neurotrophic factor levels in depressed patients: a preliminary study. Prog Neuropsychopharmacol Biol Psychiatry. 2005; 29:261-5.

[12] Gonul AS, Akdeniz F, Taneli F, Donat O, Eker Ç, Vahip S. Effect of treatment on serum brain-derived neurotrophic factor levels in depressed patients. Eur Arch Psychiatry Clin Neurosci. 2005; 255:381-6.

[13] Manfredini R, Caracciolo S, Salmi R, Boari B, Tomelli A, Gallerani $\mathrm{M}$. The association of low serum cholesterol with depression and suicidal behaviours: new hypotheses for the missing link. J Int Med Res. 2000; 28:247-57.

[14] Pfrieger FW. Role of cholesterol in synapse formation and function. Biochim Biophys Acta. 2003; 1610:271-80.

[15] Takamori S, Holt M, Stenius K, Lemke EA, Grønborg M, Riedel D, et al. Molecular anatomy of a trafficking organelle. Cell. 2006; 127:831-46.

[16] Suzuki S, Numakawa T, Shimazu K, Koshimizu H, Hara T, Hatanaka $\mathrm{H}$, et al. BDNF-induced recruitment of TrkB receptor into neuronal lipid rafts. J Cell Biol. 2004; 167:1205-15.

[17] Suzuki S, Kiyosue K, Hazama S, Ogura A, Kashihara M, Hara $\mathrm{T}$, et al. Brain-derived neurotrophic factor regulates cholesterol metabolism for synapse development. J Neurosci. 2007; 27:6417-27.

[18] Goldstein JL, DeBose-Boyd RA, Brown MS. Protein sensors for membrane sterols. Cell. 2006; 124:35-46.

[19] Thiele C, Hannah MJ, Fahrenholz F, Huttner WB. Cholesterol binds to synaptophysin and is required for biogenesis of synaptic vesicles. Nat Cell Biol. 2000; 2:42-9.

[20] Zhang J, McKeown RE, Hussey JR, Thompson SJ, Woods JR, Ainsworth BE. Low HDL cholesterol is associated with suicide attempt among young healthy women: the Third National Health and Nutrition Examination Survey. J Affect Disord. 2005; 89:25-33.

[21] Kim Y-K, Myint A-M. Clinical application of low serum cholesterol as an indicator for suicide risk in major depression. J Affect Disord. 2004; 81:161-6.

[22] Deisenhammer EA, Kramer-Reinstadler K, Liensberger D, Kemmler G, Hinterhuber H, Fleischhacker WW. No evidence for an association between serum cholesterol and the course of depression and suicidality. Psychiatry Res. 2004; 121:253-61.

[23] Huang T-L, Wu S-C, Chiang Y-S, Chen J-F. Correlation between serum lipid, lipoprotein concentrations and anxious state, depressive state or major depressive disorder. Psychiatry Res. 2003; 118:147-53.

[24] Baek JH, Kang E-S, Fava M, Mischoulon D, Nierenberg AA, Yu B-H, et al. Serum lipids, recent suicide attempt and recent suicide status in patients with major depressive disorder. Prog Neuropsychopharmacol Biol Psychiatry. 2014; 51:113-8.

[25] Park Y-M, Lee B-H, Lee S-H. The association between serum lipid levels, suicide ideation, and central serotonergic activity in patients with major depressive disorder. J Affect Disord. 2014; 159:62-5.

[26] Liang Y, Yan Z, Cai C, Jiang H, Song A, Qiu C. Association between lipid profile and depressive symptoms among Chinese older people: mediation by cardiovascular diseases? Int J Behav Med. 2014; 21:590-6.

[27] Beydoun M, Beydoun H, Dore G, Fanelli-Kuczmarski M, Evans M, Zonderman A. Total serum cholesterol, atherogenic indices and their longitudinal association with depressive symptoms among US adults. Transl Psychiatry. 2015; 5:e518. doi: 10.1038/tp.2015.4. 